\title{
SPATIAL THEORY FOR ALGEBRAS OF UNBOUNDED OPERATORS. II
}

\author{
A. INOUE AND K. TAKESUE
}

\begin{abstract}
In the previous paper [6], we have studied the spatial theory of $O_{p}^{*}$ algebras with a strongly cyclic vector. In this paper, we will investigate the spatial theory between $O_{p}^{*}$-algebras induced by a positive invariant sesquilinear form, which contains the former result.
\end{abstract}

1. Introduction. The spatial theory of von Neumann algebras has been investigated in detail, but the study for unbounded operator algebras seems to be hardly done except in [6 and 7].

In this paper we will continue the study in a more general framework which contains the former result [6].

In $\S 2$, we will introduce some notions which are used in this paper, for example, $O_{p}^{*}$-algebra, positive invariant sesquilinear form and so on.

In $\S 3$, we will consider a generalization of strongly cyclic vector for an $O_{p}^{*}$-algebra, which we call a strongly cyclic vector representation.

In $\$ 4$, we will investigate the spatial theory between $O_{p}^{*}$-algebras induced by a positive invariant sesquilinear form.

2. Preliminaries. Let $\mathscr{D}$ be a dense subspace of a Hilbert space $\mathcal{H}$. By $\mathscr{L}(\mathscr{Q})$, we denote the set of all closed operators of $\mathscr{D}$ into $\mathscr{Q}$. By $\mathfrak{L}^{+}(\mathscr{D})$ we denote the set of all $A \in \mathcal{Q}(\mathscr{D})$ with $\mathscr{D}\left(A^{*}\right) \supset \mathscr{D}$ and $A^{*} \cup \mathcal{D} \subset \mathcal{D}$, where $\mathscr{D}\left(A^{*}\right)$ denotes the domain of the adjoint operator $A^{*}$. When we put $A^{+}=A^{*} / \mathcal{O D}$, the map $A \in \mathrm{L}^{+}(\mathcal{D} \mathrm{D}) \rightarrow A^{+} \in \mathrm{L}^{+}$(बD) becomes an involution, and $\mathfrak{L}^{+}(\mathcal{D})$ is a *-algebra with the usual operation. For a subalgebra $\mathfrak{A}$ of $\mathfrak{L}(\mathscr{Q}),(\mathfrak{A}, \mathscr{D})$ is called an $O_{p}$-algebra and for a $*$-subalgebra $\mathfrak{A}$ of $\mathfrak{L}^{+}(\mathcal{O}),(\mathfrak{A}, \mathcal{O})$ is called an $O_{p}^{*}$-algebra. We note that an $O_{p}^{*}$-algebra is an $O_{p}$-algebra.

Let $(\mathfrak{A}, \mathcal{Q})$ be an $O_{p}$-algebra. Then we define seminorms $\|\cdot\|_{A}$ on oD by $\|\xi\|_{A}=$ $\|A \xi\|$ for $A \in \mathfrak{A}_{I}$ and $\xi \in \mathscr{O}$, where $\mathfrak{A}_{I}$ denotes the algebra obtained by adjoining an identity operator to $\mathfrak{A}$. The locally convex topology on of defined by the seminorms $\left\{\|\cdot\|_{A} ; A \in \mathfrak{A}_{I}\right\}$ is called the induced topology $t_{\mathfrak{H}}$. If $\underline{Q}(\mathfrak{U}) \equiv$ $\cap_{A \in \mathscr{I}}\left(\mathscr{D}(\bar{A})=\mathscr{O},(\mathfrak{A}, \mathscr{Q})\right.$ is called closed. Let $(\mathfrak{A}, \mathscr{D})$ be an $O_{p}^{*}$-algebra. An $O_{p}^{*}$-algebra $(\mathfrak{H}, \mathscr{Q})$ satisfying $\bigcap_{A \in \mathscr{H}} \mathscr{Q}\left(A^{*}\right)=\mathscr{Q}$ is called selfadjoint.

Let $\mathcal{Q}$ be a *-algebra which does not necessarily possess an identity. A sesquilinear form $\phi$ on $Q$ is called positive invariant if

$$
\phi(x, x) \geqslant 0 \text { and } \phi(a x, y)=\phi\left(x, a^{*} y\right)
$$

Received by the editors December 29, 1981

1980 Mathematics Subject Classification. Primary 47D40, 46K10; Secondary 46L10.

-1983 American Mathematical Society $0002-9939 / 82 / 0000-0652 / \$ 02.50$ 
for each $a, x, y \in Q$. Let $\phi$ be a positive invariant sesquilinear form on a $*$-algebra $Q$. Then we obtain the following quartet $\left(\pi_{\phi}(\mathscr{Q}), \mathscr{D}_{\phi}, \lambda_{\phi}, \mathcal{H}_{\phi}\right)$ by the well-known GNS-construction of $\phi$, where $\mathscr{\mathscr { D }}_{\phi}$ is a dense subspace of a Hilbert space $\mathcal{H}_{\phi}$, $\left(\pi_{\phi}(Q), \mathscr{D}_{\phi}\right)$ is a closed $O_{p}^{*}$-algebra and $\lambda_{\phi}$ is a linear map of $Q$ into $\mathscr{D}_{\phi}$ (note: $\lambda_{\phi}(\mathscr{Q})$ is dense in $\mathscr{D}_{\phi}$ with respect to the induced topology $\left.t_{\pi_{\phi}(Q)}\right)$ satisfying $\lambda_{\phi}(a x)=$ $\pi_{\phi}(a) \lambda_{\phi}(x)$ for each $a, x \in \mathbb{Q}$. We put

$$
\mathscr{D}\left(\pi_{\phi}^{*}\right)=\bigcap_{x \in \mathcal{Q}} \mathscr{D}\left(\pi_{\phi}(x)^{*}\right), \quad \pi_{\phi}^{*}(x) \xi=\pi_{\phi}\left(x^{*}\right)^{*} \xi,
$$

for each $x \in \mathbb{Q}$ and $\xi \in \mathscr{Q}\left(\pi_{\phi}^{*}\right)$. Then $\left(\pi_{\phi}^{*}(\mathbb{Q}), \mathscr{D}\left(\pi_{\phi}^{*}\right)\right)$ is an $O_{p}$-algebra. We put

$$
\mathcal{D}\left(\pi_{\phi}^{* *}\right)=\bigcap_{x \in \mathbb{Q}} \mathcal{D}\left(\pi_{\phi}^{*}(x)^{*}\right), \quad \pi_{\phi}^{* *}(x) \xi=\pi_{\phi}^{*}\left(x^{*}\right)^{*} \xi,
$$

for each $x \in \mathbb{Q}$ and $\xi \in \mathscr{D}\left(\pi_{\phi}^{* *}\right)$. Then $\left(\pi_{\phi}^{* *}(\mathcal{Q}), \mathscr{D}\left(\pi_{\phi}^{* *}\right)\right)$ is an $O_{p}^{*}$-algebra. The form $\phi$ is called Riesz if $\mathscr{D}\left(\pi_{\phi}^{*}\right)=\mathscr{O}_{\phi}$.

3. Strongly cyclic vector representations. In the previous paper [6], we investigated the spatial theory of $O_{p}^{*}$-algebras with a strongly cyclic vector. It will be natural that we consider a generalization, which is defined in the following, of the notion of strongly cyclic vector for an $O_{p}^{*}$-algebra which does not necessarily have an identity.

Let $(\mathfrak{A}, \mathscr{D})$ be a closed $O_{p}^{*}$-algebra which does not necessarily have an identity and let $\lambda$ be a linear mapping of $\mathfrak{A}$ into $\mathscr{Q}$. Then $\lambda$ is called a vector representation if $\lambda(A B)=A \lambda(B)$ holds for each $A, B \in \mathfrak{A}$. In particular, if $\lambda(\mathfrak{A})$ is dense in $\mathscr{L}$ with respect to the induced topology $t_{\mathfrak{U}}$, then $\lambda$ is called a strongly cyclic vector representation of $\mathfrak{A}$ into $\mathscr{D}$. We now consider the following two classes $\mathcal{X}, \mathscr{Y}$ :

$$
\begin{gathered}
\mathcal{X}=\left\{\begin{array}{l|l}
(\mathbb{Q}, \phi) & \begin{array}{l}
\mathbb{Q} \text { is a } * \text {-algebra and } \phi \text { is a positive invariant } \\
\text { sesquilinear form on } \mathcal{Q} \text { such that } \pi_{\phi} \text { is faithful }
\end{array}
\end{array}\right\}, \\
\mathcal{Y}=\left\{\begin{array}{ll}
(\mathfrak{A}, \mathscr{Q}, \lambda) & \begin{array}{l}
\mathfrak{A} \text { is a closed } O_{p}^{*} \text {-algebra on a pre-Hilbert space } \mathscr{D} \\
\text { and } \lambda \text { is a strongly cyclic vector representation } \\
\text { of } \mathfrak{A} \text { into } \mathscr{Q}
\end{array}
\end{array}\right\} .
\end{gathered}
$$

We introduce an equivalence relation on $\mathscr{Y}$. Let $(\mathscr{A}, \mathscr{Q}, \lambda)$ and $(\mathcal{L}, \mathcal{E}, \mu)$ be elements of $\mathcal{Y}$. Then $(\mathfrak{A}, \mathscr{D}, \lambda)$ and $(\mathcal{L}, \mathcal{E}, \mu)$ are called equivalent if there exists a unitary transform $U$ of $\mathscr{D})$ onto $\mathcal{E}$ such that $U \mathfrak{A} U^{+}=\mathcal{E}$ and $U \lambda(A)=\mu\left(U A U^{+}\right)$for each $A \in \mathfrak{A}$. In this context, we have the following Proposition.

Proposition. There exists a map $F$ of $\mathscr{X}$ into $\mathcal{Y}$ such that for each $(\mathfrak{A}, \mathcal{L}, \lambda) \in \mathcal{Y}$ we can assure the existence of an element $(Q, \phi)$ of $\mathcal{X}$ whose image by $F$ is equivalent to $\left(\mathfrak{U}, \varrho^{2}, \lambda^{\prime}\right)$.

Proof. Let $(\Theta, \phi)$ be an element of $\mathcal{X}$. Naturally we have a closed $O_{p}^{*}$-algebra $\left(\pi_{\phi}\left(\mathcal{G}^{\prime}\right), \mathcal{O}_{\phi}\right)$ by GNS-construction of $\phi$. We now define a map $\lambda$ of $\pi_{\phi}(Q)$ into $\mathcal{O Q}_{\phi}$, putting $\lambda\left(\pi_{\phi}(a)\right)=\lambda_{\phi}(a)$ for each $a \in Q$ which is well defined since $\pi_{\phi}$ is faithful. Clearly $\left(\pi_{\phi}(\mathscr{Q}), \mathscr{D}_{\phi}, \lambda\right)$ is an element of $\mathcal{Y}$. We now define a map $F$ of $\mathcal{X}$ into $\mathcal{G}$, putting $F((Q, \phi))=\left(\pi_{\phi}(Q), \mathscr{D}_{\phi}, \lambda\right)$. In the following we will show the latter part of this Proposition. Let $(\mathfrak{A}, \mathscr{D}, \lambda)$ be an element of $\mathscr{Q}$. We now define a positive 
invariant sesquilinear form $\phi$ on $\mathcal{H}$, putting $\phi(x, y)=(\lambda(x) \mid \lambda(y))$ for each $x, y \in$ $\mathfrak{A}$. We consider the closed $O_{p}^{*}$-algebra $\left(\pi_{\phi}(\mathscr{H}), \mathscr{D}_{\phi}\right)$ by GNS-construction of $\phi$. Since $\phi(x, y)=(\lambda(x) \mid \lambda(y))=\left(\lambda_{\phi}(x) \mid \lambda_{\phi}(y)\right)$ for each $x, y \in \mathfrak{U}$, there exists a unitary transform $U$ of $\cup \mathcal{D}$ onto $\mathcal{Q}_{\phi}$ such that $U \lambda(x)=\lambda_{\phi}(x)$ for each $x \in \mathfrak{A}$. Then we have

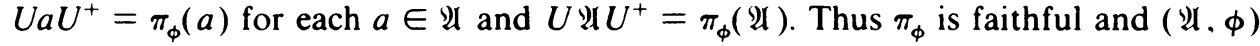
$\in \mathfrak{X}$. When we put $F((\mathfrak{H}, \phi))=\left(\pi_{\phi}(\mathfrak{H}), \cup_{\phi}, \mu\right)$, by the definition of $F, \mu$ is a map of $\pi_{\phi}(\mathscr{H})$ into $\mathscr{H}_{\phi}$ defined by $\mu\left(\pi_{\phi}(a)\right)=\lambda_{\phi}(a)$ for each $a \in \mathscr{H}$. Thus we have $U \lambda(a)=$ $\lambda_{\phi}(a)=\mu\left(\pi_{\phi}(a)\right)=\mu\left(U a U^{+}\right)$for each $a \in \mathscr{H}$. Therefore $(\mathfrak{H}, \cdot \mathfrak{l}, \lambda)$ is equivalent to $\left(\pi_{\phi}(\mathscr{U}), \mathscr{N}_{\phi}, \mu\right)=F((\mathfrak{H}, \phi))$. This completes the proof.

By the above consideration, we see that the investigation of $(\mathcal{H}, \cdots, \lambda) \in \forall$ is equivalent to that of $\left(G^{\prime}, \phi\right) \in x^{\prime}$.

4. Spatial theory. First of all, we introduce some notation to clarify the object with which we will now deal for spatial theory. Let $A^{\prime}, 4 B$ be $*$-algebras which do not necessarily have an identity. By $I\left(\mathcal{U}^{\prime}, \varphi^{\prime}\right)$ we denote the set of all *-isomorphisms of $\mathcal{C}^{\prime}$ onto $\varphi_{h}$. Let $\phi$ (resp. $\psi$ ) be a positive invariant sesquilinear form on $Q$ (resp. 6 ). By $I\left(\left(\mathcal{C}^{\prime}, \phi\right),\left(()_{1}, \psi\right)\right)$ we denote the set of all $\alpha \in I\left(\mathcal{Q}^{\prime}, G B\right)$ satisfying $\alpha\left(\operatorname{ker} \pi_{\phi}\right)=\operatorname{ker} \pi_{\psi}$. Let $\left(\pi_{\phi}\left(\mathcal{G}^{\prime}\right), \mathcal{\cup D}_{\phi}, \lambda_{\phi}\right)\left(\right.$ resp. $\left.\left(\pi_{\psi}\left(\sigma_{B}\right), \sigma_{\psi}, \lambda_{\psi}\right)\right)$ be the closed $O_{p}^{*}$-algebra by the GNSconstruction of $\phi$ (resp. $\psi)$. Then a $*$-isomorphism $\alpha_{\psi \phi}$ of $\pi_{\phi}(Q)$ onto $\pi_{\psi}(G)$ is defined by

$$
\alpha_{\psi \phi}\left(\pi_{\phi}(x)\right)=\pi_{\psi}(\alpha(x))
$$

for each $x \in \mathbb{Q}$.

In this paper, we will investigate when $*$-isomorphisms $\alpha_{\psi \phi}$ gained in this manner can be spatially realized. We note that this setting contains the spatial problem between the closed $O_{p}^{*}$-algebras with a strongly cyclic vector representation by the consideration of $\S 3$.

THEOREM 4.1. Let $\phi$ and $\psi$ be the positive invariant sesquilinear forms on *-algebras $\mathcal{H}^{\prime}$ and $\cup^{\prime}$ respectively, and let $\alpha$ be an element of $I((Q, \phi),(\mathscr{B}, \psi))$.

(1) The following statements are equivalent.

(1.1) There exists an isometry $U$ of $\mathcal{D}_{\psi}$ into $\left.{ }^{\circ}\right)\left(\pi_{\phi}^{*}\right)$ such that $U \alpha_{\psi \phi}\left(\pi_{\phi}(x)\right)=\pi_{\phi}^{*}(x) U$ for each $x \in \mathcal{Q}$.

(1.2) There exists a linear map $\mu$ of $Q$ into $\cup 1)\left(\pi_{\phi}^{*}\right)$ such that $\mu(a x)=\pi_{\phi}^{*}(a) \mu(x)$ and $\left(\lambda_{\psi}(\alpha(x)) \mid \lambda_{\psi}(\alpha(y))\right)=(\mu(x) \mid \mu(y))$ for each $a, x, y \in \mathbb{Q}$.

(2) Under the condition of (1), the following statements hold.

(2.1) There exists an isometry $U$ of $\mathscr{D}_{\psi}$ into $\mathcal{D}\left(\pi_{\phi}^{*}\right)$ such that $\alpha_{\psi \phi}\left(\pi_{\phi}(x)\right)=U^{*} \pi_{\phi}^{*}(x) U$ for each $x \in \mathbb{Q}$. Moreover, $U^{*} \mathcal{D}\left(\pi_{\phi}^{* *}\right) \subset \mathcal{D}\left(\pi_{\psi}^{*}\right)$ and $\pi_{\psi}^{*}(\alpha(x)) U^{*} \xi=U^{*} \pi_{\phi}^{* *}(x) \xi$ for each $x \in \mathcal{G}^{\prime}$ and $\xi \in \mathcal{Q}\left(\pi_{\phi}^{* *}\right)$.

(2.2) If $\pi_{\psi}^{* *}=\pi_{\psi}^{*}$ (in particular, if $\psi$ is a Riesz form), we have $\bar{U} U^{*} \in \pi_{\phi}(Q \mathcal{Q})^{\prime}$, where $\pi_{\phi}\left(\mathcal{U}^{2}\right)^{\prime}$ denotes the set of all bounded operators $A$ on $\mathcal{K}_{\phi}$ satisfying $\left(A \pi_{\phi}(x) \xi \mid \eta\right)$ $=\left(A \xi \mid \pi_{\phi}\left(x^{*}\right) \eta\right)$ for each $x \in \mathbb{Q}$ and $\xi, \eta \in \mathcal{D}_{\phi}$.

(2.3) Suppose that $\pi_{\psi}^{*}=\pi_{\psi}, \pi_{\phi}(Q)^{\prime}=\mathrm{CI}$ and $\mu(\Theta) \subset \mathscr{D}_{\phi}$. Then $U$ is a unitary transform of $\mathscr{D}_{\psi}$ onto $\mathscr{D}_{\phi}$ and $\phi$ is a Riesz form. Therefore, in this case, we have $\alpha_{\psi \phi}\left(\pi_{\phi}(x)\right)=U^{+} \pi_{\phi}(x) U$ for each $x \in \mathbb{Q}$, where $U^{+}$denotes the restriction of $U^{*}$ to Ui $\phi_{\phi}$. 
Proof. (1) (1.1) $\Rightarrow$ (1.2). Putting $\mu(x)=U \lambda_{\psi}(\alpha(x))$ for each $x \in \mathcal{Q}$, we easily see that this map $\mu$ satisfies all the conditions of (1.2).

$(1.2) \Rightarrow(1.1)$. By the assumption, there exists an isometry $U$ of $0 D_{\psi}$ into $: \mathcal{H}_{\phi}$ such that $U \lambda_{\psi}(\alpha(x))=\mu(x)$ for each $x \in \mathcal{C}^{\prime}$. Let $\xi$ be an arbitrary element of ${ }^{\circ} D_{\psi}$. Then there exists a net $\left\{y_{i}\right\}$ in $\mathcal{C}^{\prime}$ such that $\lambda_{\psi}\left(\alpha\left(y_{i}\right)\right)$ converges to $\xi$ in the induced topology $t_{\pi_{\downarrow}(\cup B)}$. For each $x, y \in(\mathcal{Q}$ we have

$$
\begin{aligned}
\left(\pi_{\phi}\left(x^{*}\right) \lambda_{\phi}(y) \mid U \xi\right) & =\lim _{i}\left(\pi_{\phi}\left(x^{*}\right) \lambda_{\phi}(y) \mid U \lambda_{\psi}\left(\alpha\left(y_{i}\right)\right)\right) \\
& =\lim _{i}\left(\pi_{\phi}\left(x^{*}\right) \lambda_{\phi}(y) \mid \mu\left(y_{i}\right)\right)=\lim _{i}\left(\lambda_{\phi}(y) \mid \pi_{\phi}^{*}(x) \mu\left(y_{i}\right)\right) \\
& =\lim _{i}\left(\lambda_{\phi}(y) \mid \mu\left(x y_{i}\right)\right)=\lim _{i}\left(\lambda_{\phi}(y) \mid U \lambda_{\psi}\left(\alpha\left(x y_{i}\right)\right)\right) \\
& =\lim _{i}\left(\lambda_{\phi}(y) \mid U \pi_{\psi}(\alpha(x)) \lambda_{\psi}\left(\alpha\left(y_{i}\right)\right)\right)=\left(\lambda_{\phi}(y) \mid U \pi_{\psi}(\alpha(x)) \xi\right) .
\end{aligned}
$$

Hence we have $U \xi \in \cup D\left(\pi_{\phi}\left(x^{*}\right)^{*}\right)$ for each $x \in \mathcal{Q}$ and thus we have $\left.U \xi \in \mathcal{Q}^{1}\right)\left(\pi_{\phi}^{*}\right)$ and $\pi_{\phi}^{*}(x) U \xi=U \pi_{\psi}(\alpha(x)) \xi$ for each $x \in \mathcal{Q}$. and $\xi \in \mathcal{D D}_{\psi}$. This shows that the isometry $U$ satisfies all the conditions of (1.1).

(2) (2.1) Let $U$ be an isometry of $\mathcal{D} \mathbb{D}_{\psi}$ into $\mathscr{D}\left(\pi_{\phi}^{*}\right)$ such that $U \boldsymbol{\alpha}_{\psi \phi}\left(\pi_{\phi}(x)\right)=\pi_{\phi}^{*}(x) U$ for each $x \in \mathbb{Q}^{*}$. It is then clear that $\alpha_{\psi \phi}\left(\pi_{\phi}(x)\right)=U^{*} \pi_{\phi}^{*}(x) U$ for each $x \in \mathbb{Q}$. For each $\xi \in$ 'D $\left(\pi_{\phi}^{* *}\right), \eta \in \mathcal{D}_{\psi}$ and $x \in \mathcal{U}^{\prime}$, we have

$$
\begin{aligned}
\left(\pi_{\psi}\left(\alpha\left(x^{*}\right)\right) \eta \mid U^{*} \xi\right) & =\left(U \pi_{\psi}\left(\alpha\left(x^{*}\right)\right) \eta \mid \xi\right)=\left(U \alpha_{\psi \phi}\left(\pi_{\phi}\left(x^{*}\right)\right) \eta \mid \xi\right) \\
& =\left(\pi_{\phi}^{*}\left(x^{*}\right) U \eta \mid \xi\right)=\left(U \eta \mid \pi_{\phi}^{* *}(x) \xi\right)=\left(\eta \mid U^{*} \pi_{\phi}^{* *}(x) \xi\right) .
\end{aligned}
$$

Hence we have $U^{*} \xi \in \mathscr{D}\left(\pi_{\psi}\left(\alpha\left(x^{*}\right)\right)^{*}\right)$ for each $x \in \mathcal{Q}$ and thus $U^{*} \xi \in \mathscr{D}\left(\pi_{\psi}^{*}\right)$ and $\pi_{\psi}^{*}(\alpha(x)) U^{*} \xi=U^{*} \pi_{\phi}^{* *}(x) \xi$.

(2.2) We now suppose that $\pi_{\psi}^{* *}=\pi_{\psi}^{*}$. Then, since $U^{*} \lambda_{\phi}(y) \in \mathcal{O}\left(\pi_{\psi}^{*}\right)=\mathcal{O} D\left(\pi_{\psi}^{* *}\right)$ for each $y \in \mathbb{U}^{*}$, it follows that for each $a, x, y \in \mathbb{Q}$

$$
\begin{aligned}
\left(\bar{U} U^{*} \pi_{\phi}(a) \lambda_{\phi}(x) \mid \lambda_{\phi}(y)\right) & =\left(\pi_{\psi}^{*}(\alpha(a)) U^{*} \lambda_{\phi}(x) \mid U^{*} \lambda_{\phi}(y)\right) \\
& =\left(U^{*} \lambda_{\phi}(x) \mid \pi_{\psi}^{*}\left(\alpha\left(a^{*}\right)\right) U^{*} \lambda_{\phi}(y)\right) \\
& =\left(U^{*} \lambda_{\phi}(x) \mid U^{*} \pi_{\phi}^{* *}\left(a^{*}\right) \lambda_{\phi}(y)\right) \\
& =\left(\bar{U} U^{*} \lambda_{\phi}(x) \mid \pi_{\phi}\left(a^{*}\right) \lambda_{\phi}(y)\right) .
\end{aligned}
$$

Hence we have $\bar{U} U^{*} \in \pi_{\phi}(\mathbb{Q})^{\prime}$.

(2.3) By the assumption, it is clear that $\bar{U}$ is a unitary transform of $\mathcal{H}_{\psi}$ onto $\mathcal{H}_{\phi}$ and $U \mathscr{D}_{\psi} \subset \mathscr{O}_{\phi}$ (since $U \mathscr{D}_{\psi}$ is the $t_{\pi_{\phi}^{*}\left(Q^{*}\right)}$-closure of $\mu(\mathbb{Q})$ ). On the other hand, by $(2.1)$ we have $U^{+} \mathscr{D}_{\phi} \subset \mathscr{D}_{\psi}$. Thus we have $U \mathscr{D}_{\psi}=\mathscr{O Q D}_{\phi}$. This shows that $U$ is a unitary transform of $\mathcal{O D}_{\psi}$ onto $\mathcal{D}_{\phi}$. Therefore we have $\alpha_{\psi \phi}\left(\pi_{\phi}(x)\right)=U^{+} \pi_{\phi}(x) U$ for each $x \in \mathbb{Q}$. It is almost clear that $\phi$ is a Riesz form. This completes the proof.

Let $\mathcal{Q}$ be a $*$-algebra and $\phi, \psi$ be positive invariant sesquilinear forms on $\mathcal{Q}$. Then $\psi$ is called $\phi$-bounded if for each $y \in \mathbb{Q}$ there exists a positive number $\gamma_{y}$ such that $|\psi(x, y)|^{2} \leqslant \gamma_{y} \phi(x, x)$ for each $x \in \mathbb{A}$. If there exists a positive number $\gamma$ such that $\psi(x, x) \leqslant \gamma \phi(x, x)$ for each $x \in \mathbb{Q}$, then $\psi$ is called $\phi$-dominated. Let $I_{b}((Q, \phi),(\mathscr{B}, \psi))\left(\right.$ resp. $\left.I_{d}((Q, \phi),(\mathscr{B}, \psi))\right)$ be the set of all $\alpha \in I((Q, \phi),(\mathscr{B}, \psi))$ 
satisfying that $\psi_{\alpha}$ is $\phi$-bounded (resp. $\phi$-dominated), where $\psi_{\alpha}$ is a positive invariant sesquilinear form on $Q$ defined by $\psi_{\alpha}(x, y)=\psi(\alpha(x), \alpha(y))$ for each $x, y \in \mathbb{Q}$.

Before proceeding with our argument, we note the following well-known fact (therefore we omit its proof).

LEMMA 4.2 (FRIEDRICH'S EXTENSION). Let 0 D be a dense linear subspace of a Hilbert space $\mathcal{H}$. Let $T_{0}$ be an operator on $\mathcal{K}$ with domain $\mathscr{D}$. Suppose that $\left(T_{0} \xi \mid \xi\right) \geqslant 0$ for each $\xi \in \mathscr{D}$. Then there exists a selfadjoint positive operator $T$ such that $T$ is an extension of $T_{0}$ and $X \mathscr{D}(T) \subset \mathscr{D}(T)$ and $X T \xi=T X \xi$ for each $X \in \mathscr{B}\left(T_{0}\right)$ and $\xi \in \mathscr{D}(T)$, where $\mathscr{D}(T)$ is the domain of $T$ and $\mathscr{B}\left(T_{0}\right)$ is the set of all bounded operators $X$ on $\mathcal{H}$ satisfying $X^{\mathscr{D}} \subset \mathscr{D}, X^{*} \mathscr{D} \subset \mathscr{D}$ and $X T_{0} \xi=T_{0} X \xi, X^{*} T_{0} \xi=T_{0} X^{*} \xi$ for each $\xi \in \mathscr{D}$.

THEOREM 4.3. Let $\alpha$ be an element of $I_{b}((Q, \phi),(\mathscr{B}, \psi))$. Suppose that $\phi$ is admissible; that is, $\pi_{\phi}(x)$ is a bounded operator for each $x \in \mathcal{Q}$. Then, $\psi$ is also admissible and there exists an isometry $U$ of $\mathcal{H}_{\psi}$ into $\mathcal{H}_{\phi}$ such that $\alpha_{\psi \phi}\left(\pi_{\phi}(x)\right)=$ $U^{*} \pi_{\phi}(x) U$ for each $x \in \mathbb{Q}$.

Proof. Putting $K_{0} \lambda_{\phi}(x)=\lambda_{\psi}(\alpha(x))$ for each $x \in \mathcal{Q}$, we define an operator $K_{0}$ with domain $\lambda_{\phi}(\mathbb{Q})$, which is well defined by the assumption. The inequality $|\psi(\alpha(x), \alpha(y))|^{2} \leqslant \gamma_{y} \phi(x, x)$ means that $\left|\left(K_{0} \lambda_{\phi}(x) \mid \lambda_{\psi}(\alpha(y))\right)\right| \leqslant \gamma_{y}^{1 / 2}\left\|\lambda_{\phi}(x)\right\|$. Hence $\lambda_{\psi}(\mathscr{B})\left(=K_{0} \lambda_{\phi}(\mathscr{Q})\right)$ is contained in $\mathscr{D}\left(K_{0}^{*}\right)$. For each $a, x, y \in \mathscr{Q}$, we have

$$
\begin{aligned}
\left(K_{0}^{*} K_{0} \pi_{\phi}(a) \lambda_{\phi}(x) \mid \lambda_{\phi}(y)\right) & =\left(\lambda_{\psi}(\alpha(a x)) \mid \lambda_{\psi}(\alpha(y))\right) \\
& =\left(\pi_{\psi}(\alpha(a)) \lambda_{\psi}(\alpha(x)) \mid \lambda_{\psi}(\alpha(y))\right) \\
& =\left(\lambda_{\psi}(\alpha(x)) \mid \pi_{\psi}\left(\alpha\left(a^{*}\right)\right) \lambda_{\psi}(\alpha(y))\right) \\
& =\left(K_{0} \lambda_{\phi}(x) \mid K_{0} \lambda_{\phi}\left(a^{*} y\right)\right) \\
& =\left(K_{0}^{*} K_{0} \lambda_{\phi}(x) \mid \pi_{\phi}\left(a^{*}\right) \lambda_{\phi}(y)\right) \\
& =\left(\pi_{\phi}(a) K_{0}^{*} K_{0} \lambda_{\phi}(x) \mid \lambda_{\phi}(y)\right)
\end{aligned}
$$

(since $\pi_{\phi}\left(a^{*}\right)$ is a bounded operator). Hence we have $K_{0}^{*} K_{0} \pi_{\phi}(a) \lambda_{\phi}(x)=$ $\pi_{\phi}(a) K_{0}^{*} K_{0} \lambda_{\phi}(x)$ for each $a, x \in \mathbb{Q}$. When we use Lemma 4.2 for $K_{0}^{*} K_{0}$, we can easily see the existence of a selfadjoint positive operator $H$ such that $H$ is an extension of $K_{0}^{*} K_{0}$ and affiliated with the von Neumann algebra $\pi_{\phi}(\mathbb{Q})^{\prime}$. For each $x, y \in \mathbb{Q}$, we have

$$
\left(H^{1 / 2} \lambda_{\phi}(x) \mid H^{1 / 2} \lambda_{\phi}(y)\right)=\left(\lambda_{\psi}(\alpha(x)) \mid \lambda_{\psi}(\alpha(y))\right) .
$$

Therefore, when we put $\mu(x)=H^{1 / 2} \lambda_{\phi}(x)$ for each $x \in \mathbb{Q}$, the map $\mu$ satisfies all the conditions of Theorem 4.1(1.2). Thus there exists an isometry $U$ of $\mathscr{D}_{\psi}$ into $\mathcal{H}_{\phi}$ such that $\alpha_{\psi \phi}\left(\pi_{\phi}(x)\right)=U^{*} \pi_{\phi}(x) U$ for each $x \in \mathbb{Q}$. On the other hand, since

$$
\pi_{\psi}(\alpha(x))=\alpha_{\psi \phi}\left(\pi_{\phi}(x)\right)=U^{*} \pi_{\phi}(x) U
$$

for each $x \in \mathscr{Q}, \pi_{\psi}(y)$ is a bounded operator for each $y \in \mathscr{B}$. Thus $\mathscr{Q}_{\psi}=\mathscr{H}_{\psi}$ and $\psi$ is admissible. This completes the proof. 
THEOREM 4.4. Let $\alpha$ be an element of $I_{d}((Q, \phi),(9, \psi))$. Suppose that $\phi$ is a Riesz form. Then there exists an isometry $U$ of $\mathscr{D}_{\psi}$ into $\mathscr{D}_{\phi}$ such that $\alpha_{\psi \phi}\left(\pi_{\phi}(x)\right)=U^{+} \pi_{\phi}(x) U$ for each $x \in \mathbb{Q}$.

Proof. By the assumption, there exists a bounded operator $K$ of $\mathcal{H}_{\phi}$ into $\mathcal{H}_{\psi}$ such that $K \lambda_{\phi}(x)=\lambda_{\psi}(\alpha(x))$ for each $x \in \mathbb{Q}$. Then we can easily see that $K^{*} K \in \pi_{\phi}(\mathbb{Q})^{\prime}$. Since $\pi_{\phi}(Q)^{\prime}$ is a von Neumann algebra, $\left(K^{*} K\right)^{1 / 2}$ is an element of $\pi_{\phi}(\mathbb{Q})^{\prime}$. Now we put $\mu(x)=\left(K^{*} K\right)^{1 / 2} \lambda_{\phi}(x)$. Then it is almost clear that $\mu$ satisfies all the conditions of Theorem 4.1(1.2). Hence there exists an isometry $U$ of $\mathscr{D}_{\psi}$ into $\mathscr{D}_{\phi}$ such that $\alpha_{\psi \phi}\left(\pi_{\phi}(x)\right)=U^{+} \pi_{\phi}(x) U$ for each $x \in \mathbb{Q}$. This completes the proof.

\section{REFERENCES}

1. J. Dixmier, Les algèbres d'opérateurs dans l'espace Hilbertian, 2é ed., Gauthier-Villars, Paris, 1969

2. S. P. Gudder and W. Scruggs, Unbounded representations of *-algebras, Pacific J. Math. 70 (1977), 369-382.

3. A. Inoue, Operator-representations and vector-representations of positive linear functionals, Fukuoka Univ. Rep. (to appear).

4. G. Lassner, Topological algebras of operators, Rep. Math. Phys. 3 (1972). 279-293.

5. R. T. Powers, Self-adjoint algebras of unbounded operators, Comm. Math. Phys. 21 (1971). 85-124.

6. K. Takesue, Spatial theory for algebras of unbounded operators, Rep. Math. Phys. (to appear)

7. A. Uhlmann, Properties of the algehra $L^{+}(D)$, JINR, Comm. E 2-8149, Dubna. 1974.

Department of Applied Mathematics. Fukuoka University, 814-01, Fukuoka, Japan

Department of Mathematics, KyUshu University, FukUoka, Japan 